\title{
Large Barocaloric Effect with High Pressure-Driving Efficiency in a Hexagonal $\mathrm{MnNi}_{0.77} \mathrm{Fe}_{0.23} \mathrm{Ge}$ Alloy
}

\author{
Qingqi Zeng(曾庆祺 $)^{1,2}$, Jianlei Shen(申建雷 $)^{1,2}$, Enke Liu(刘恩克 $)^{1,3 *}$, Xuekui Xi(郗学奎 $)^{1}$, \\ Wenhong Wang(王文洪 $)^{1,3}$, Guangheng Wu(吴光恒 $)^{1}$, and Xixiang Zhang(张西祥 $)^{4}$ \\ ${ }^{1}$ Institute of Physics, Chinese Academy of Sciences, Beijing 100190, China \\ ${ }^{2}$ University of Chinese Academy of Sciences, Beijing 100049, China \\ ${ }^{3}$ Songshan Lake Materials Laboratory, Dongguan 523808, China \\ ${ }^{4}$ Physical Science and Engineering Division (PSE), King Abdullah University of Science and Technology (KAUST),
} Thuwal 23955-6900, Saudi Arabia

(Received 22 April 2020; accepted 10 May 2020; published online 21 June 2020)

\begin{abstract}
The hydrostatic pressure is expected to be an effective knob to tune the magnetostructural phase transitions of hexagonal MM'X alloys ( $\mathrm{M}$ and $\mathrm{M}$ ' denote transition metals and $\mathrm{X}$ represents main group elements). We perform magnetization measurements under hydrostatic pressure on an MM'X martensitic $\mathrm{MnNi}_{0.77} \mathrm{Fe}_{0.23} \mathrm{Ge}$ alloy. The magnetostructural transition temperature can be efficiently tuned to lower temperatures by applying moderate pressures, with a giant shift rate of $-151 \mathrm{~K} / \mathrm{GPa}$. A temperature span of $30 \mathrm{~K}$ is obtained under the pressure, within which a large magnetic entropy change of $-23 \mathrm{~J} \cdot \mathrm{kg}^{-1} \mathrm{~K}^{-1}$ in a field change of $5 \mathrm{~T}$ is induced by the mechanical energy gain due to the large volume change. Meanwhile, a decoupling of structural and magnetic transitions is observed at low temperatures when the martensitic transition temperature is lower than the Curie temperature. These results show a multi-parameter tunable caloric effect that benefits the solid-state cooling.
\end{abstract}

PACS: $61.50 . K s, 63.70 .+\mathrm{h}, 75.30 . \mathrm{Sg} \quad$ DOI: $10.1088 / 0256-307 \mathrm{X} / 37 / 7 / 076101$

In recent years, there has been great interest in hexagonal MM'X (M and M' denote transition metals and $\mathrm{X}$ represents main group elements) alloys due to the realization of magnetostructural transition, ${ }^{[1]}$ magnetocaloric effects (MCE), ${ }^{[2,3]}$ negative thermal expansion $^{[4]}$ and magneto-strain ${ }^{[5]}$ near room temperature in these systems. The hexagonal MM'X alloy $\mathrm{MnNi}_{0.77} \mathrm{Fe}_{0.23} \mathrm{Ge}$ was reported as a system with martensitic and magnetic transitions coupled together, which occurs at about $266 \mathrm{~K}$ on cooling from a paramagnetic $(\mathrm{PM})$ parent phase with the $\mathrm{Ni}_{2}$ In-type structure to ferromagnetic (FM) martensite phase with the TiNiSi-type structure. ${ }^{[1]}$ With the coupling of magnetic and structural degrees of freedom, a large $\mathrm{MCE}$ was reported on the transition. As we know, the MCE is intensively studied due to its applications in magnetic refrigeration, thermomagnetic motors, controlling of drug delivery, and so on. ${ }^{[6-8]}$ It is desirable to find external parameters to tune or enhance the MCE. For a hexagonal MM'X family, there is a remarkable character, i.e., giant volume expansion with values of $2-5 \%$ during the structural transition upon cooling. ${ }^{[1,3,9]}$ A hydrostatic pressure can drive this transition to lower temperatures as the mechanical energy gain $(\Delta P \cdot \Delta V)$ in the total Gibbs freedom energy tends to shift the equilibrium point of the phase transition. Thus, besides the chemical substitution, ${ }^{[10,11]}$ vacancy, ${ }^{[12,13]}$ magnetic field ${ }^{[14]}$ and strain, ${ }^{[15]}$ the hydrostatic pressure is widely used ${ }^{[10,11,16-21]}$ to tune a phase transition. In fact, as a clean means the pressure can be used to induce many physical behaviors. ${ }^{[22-26]}$ The studies on MCE include not only a wide temperature range but also a shift in transition temperature by external parameters, such as hydrostatic pressure, which can tune the transition to the required working temperature. ${ }^{21]}$

In this study, we investigate the effect of hydrostatic pressure on magnetism, martensitic transition and MCE based on a hexagonal MM'X alloy $\mathrm{MnNi}_{0.77} \mathrm{Fe}_{0.23} \mathrm{Ge}$. The magnetostructural transition temperature can be efficiently tuned to lower temperatures, with a driving efficiency of $-151 \mathrm{~K} / \mathrm{GPa}$. A temperature span of $30 \mathrm{~K}$ is obtained under the pressure, within which a magnetic entropy change of about $-23 \mathrm{~J} \cdot \mathrm{kg}^{-1} \mathrm{~K}^{-1}$ for a magnetic field change of $5 \mathrm{~T}$ is observed. A decoupling of structural and magnetic transitions occurs when the martensitic transition temperature is lower than the Curie temperature of parent phase at higher pressures. These results indicate that the caloric effect of this system can be tuned by multiple external parameters.

Polycrystalline ingots were prepared by arc melting high-purity metals under argon atmosphere. The ingots were then annealed at $1123 \mathrm{~K}$ for five days and slowly cooled at a rate of $1 \mathrm{~K} / \mathrm{min}$ to room temperature to avoid stress in samples. The structure of measured sample was examined by x-ray diffraction (XRD) using $\mathrm{Cu} K_{\alpha}$ radiation. Magnetization measurements were performed using a Quantum Design superconducting quantum interference device vibrating sample magnetometer (SQUID-VSM) equipped with pressure cell in fields up to $5 \mathrm{~T}$ in the temperature range of $5-300 \mathrm{~K}$ and for pressures up to $1.280 \mathrm{GPa}$. Daphne 7373 was used as the pressure transmitting medium. In order to determine the pressure in the pressure cell more accurately, a piece of $\mathrm{Pb}$ was loaded together with the proper size bulk sample as the super-

Supported by the National Natural Science Foundation of China (Grant No. 51722106), the National Key R\&D Program of China (Grant No. 2019YFA0704904), Users with Excellence Program of Hefei Science Center CAS (Grant No. 2019HSC-UE009), and Fujian Institute of Innovation, Chinese Academy of Sciences.

*Corresponding author. Email: ekliu@iphy.ac.cn

(C) 2020 Chinese Physical Society and IOP Publishing Ltd 
conducting transition temperature under certain pressure for $\mathrm{Pb}$ is known. ${ }^{[27]}$
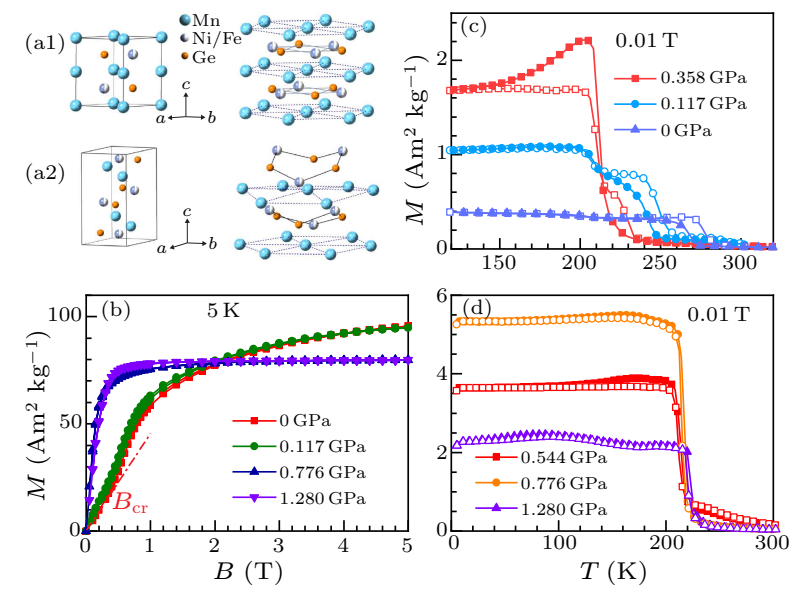

Fig. 1. (a1) and (a2) Primitive cell and a structure sketch of hexagonal parent and orthorhombic martensite phases. (b) Magnetic field dependence of the magnetization $(M-$ $H$ ) curves at $5 \mathrm{~K}$ under different pressures. (c) and (d) Temperature dependence of the magnetization $(M-T)$ in a magnetic field of $0.01 \mathrm{~T}$ under different pressures. The solid and open symbols represent the cooling and heating process, respectively.

At ambient pressure, $\mathrm{MnNi}_{0.77} \mathrm{Fe}_{0.23} \mathrm{Ge}$ undergoes a martensitic transition at about $266 \mathrm{~K}$ according to the previous report. ${ }^{[1]}$ The structures of hightemperature parent and low-temperature martensite phase are shown in Figs. 1(a1) and 1(a2). The parent phase crystallizes in a $\mathrm{Ni}_{2}$ In-type hexagonal structure with a space group of $P 6_{3} / m m c$ (194) while the martensitic phase crystallizes in a TiNiSi-type orthorhombic phase with Pnma (62). ${ }^{[28-30]}$ The volume change during the transition is about $2.7 \% .^{[1]}$ In the right panel of Figs. 1(a1) and 1(a2) we sketch the atom position relation of two phases. The structural transition occurs via distortions of $\mathrm{Ni} / \mathrm{Fe}-\mathrm{Ge}$ hexagonal rings and $\mathrm{Mn}-\mathrm{Mn}$ zigzag chains. ${ }^{[3]}$ Magnetic field dependence of the magnetization $(M-H)$ curves at $5 \mathrm{~K}$ under several pressures are shown in Fig. 1(b). Metamagnetic behavior occurs at about $0.4 \mathrm{~T}$ under low pressures ( 0 and $0.117 \mathrm{GPa}$ ), which is also consistent with the previous report. ${ }^{[1]}$ The martensite phase in the Mn-Ni-Fe-Ge system will form a spiral antiferromagnetic (AFM) structure under zero field at low temperature. ${ }^{[1,2]}$ In addition, the $M-H$ in this system shows metamagnetic transition from spiral AFM to FM at $5 \mathrm{~K}$. In a different fashion, the $M-H$ curves under 0.776 and $1.280 \mathrm{GPa}$ show FM behavior of the parent phase, indicating the suppression of martensitic transition by high pressure. Martensite phase exhibits higher saturation field and magnetization than those of the parent phase. The former characteristic may be due to the higher anisotropy in the martensite phase. The different saturation magnetization is related to the different distances between neighboring $\mathrm{Mn}$ atoms in the two phases. ${ }^{[18,31,32]}$ However, the pressure-induced changes in magnetization in both the phases (parent and martensite) are small, suggesting a minor variations of the exchange interaction, which may be attributed to a slight modification of the electronic density of states at the Fermi level under the present pressures. ${ }^{[11]}$

The variations of magnetization and martensitic transition behaviors under different pressures are depicted by temperature dependence of the magnetization $(M-T)$ in a magnetic field of $0.01 \mathrm{~T}$, as shown in Figs. $1(\mathrm{c})$ and $1(\mathrm{~d})$. The $M-T$ curves under 0 and $0.117 \mathrm{GPa}$ show clear temperature hysteresis, which is associated with the forward and reverse martensitic transition temperatures, indicating a first-order nature of the transition. The martensitic transition temperature is determined by the extreme point of the first order differential of the $M-T$ curve. The forward martensitic transition temperature $\left(T_{\mathrm{M}}\right)$ at ambient pressure is $267 \mathrm{~K}$, which is consistent with the previous report. ${ }^{[1]}$ At $T_{\mathrm{M}}$, it is a magnetostructural transition, the parent phase in PM state transforms to the martensite phase in FM order. There is a magnetization change without hysteresis around $209 \mathrm{~K}$ under $0.117 \mathrm{GPa}$, which should be the Curie temperature of residual parent phase. The change in the magnetization between parent and martensite phases becomes larger with increasing pressure. Such an increase is a consequence of the lower martensitic transition temperature, at which the magnetization of FM martensite increases.

The $M-T$ curve under $0.358 \mathrm{GPa}$ is slightly complicated due to the near decoupling of structural and magnetic phase transitions. When the temperature decreases, one can see the Curie temperature of parent phase $\left(T_{\mathrm{C}}^{\mathrm{A}}\right)$ and entangled martensitic transition temperature at about $212 \mathrm{~K}$. However, due to the lager span of structural phase transition than a magnetic one, we can still see a "tail" of the martensitic transition. Thus there is a decrease of magnetization soon after $T_{\mathrm{C}}^{\mathrm{A}}$ and $T_{\mathrm{M}}$ due to the lower magnetization of martensite compared to the parent phase in a low magnetic field of $0.01 \mathrm{~T}$. On the heating process, the first drop of magnetization is associated with the Curie temperature of residual parent phase, while the second drop at about $228 \mathrm{~K}$ is due to the reverse martensitic transition from martensite to PM parent phase. The $M-T$ curves show only a transition without hysteresis above $0.544 \mathrm{GPa}$ (Fig. 1(d)), indicating the occurrence of the suppression of martensitic transition by higher pressures, which is consistent with the above analysis about the $M-H$ curves. The martensitic transition is shifted to lower temperatures by pressure and finally vanishes at about $210 \mathrm{~K}$. As this temperature is relatively high, there should be other factors that suppress the structural transition besides the high pressure.

The variation of magnetic order of the parent phase should be one reason. It seems that the martensitic transition will vanish when the FM transition of parent phase happens first during temperature decreasing. This suppression behavior has been observed in other MM'X compounds. ${ }^{[1,9,12]}$ The interpretation could be that the parent phase is more stable at the FM state than the PM one. Thus the energy barrier between the FM parent and martensite phases becomes difficult to overcome. In the present case, this suppression behavior occurs under the condition of the hydrostatic pressure loading. Note that the sudden drops of magnetization at very low temperatures in Fig. 1(d) are associated with the superconducting 
transitions of $\mathrm{Pb}$ as a pressure probe.

Figure 2(a) shows the pressure dependence of martensitic transition temperature and Curie temperature of the parent phase. $T_{\mathrm{M}}$ and $T_{\mathrm{C}}^{\mathrm{A}}$ are determined by the extreme points of the first order differential of the $M-T$ curve. The large error bars under 0.321 and $0.330 \mathrm{GPa}$ are due to the entanglement of the structural and magnetic transitions. As one can see, $T_{\mathrm{M}}$ decreases while $T_{\mathrm{C}}^{\mathrm{A}}$ increases with increasing pressure. An increasing pressure leads to a shrinking of the cell volume and enhanced exchange interactions between moments on $\mathrm{Mn}$ atoms, which result in the rising of $T_{\mathrm{C}}^{\mathrm{A}}$. The pressure dependence of Curie temperature shows the similar behavior to the MnCoGe system. ${ }^{[35]}$ The application of hydrostatic pressure stabilizes the hexagonal parent phase because there is a big expansion of the volume during the martensitic structural transition upon cooling, which is common in hexagonal MM'X alloys. ${ }^{[3]}$ Pressures above $0.544 \mathrm{GPa}$ eventually suppress the martensitic transition.

The shift with pressure of $T_{\mathrm{M}}$ is approximately calculated to be $-151 \mathrm{~K} / \mathrm{GPa}$ in the $\mathrm{MnNi}_{0.77} \mathrm{Fe}_{0.23} \mathrm{Ge}$ system, where $d T_{\mathrm{t}} / d P=\left[T_{\mathrm{M}}(0.358 \mathrm{GPa})-\right.$ $\left.T_{\mathrm{M}}(0 \mathrm{GPa})\right] / 0.358 \mathrm{GPa}$. Figure $2(\mathrm{~b})$ compares the shift of $T_{\mathrm{M}}$ or $T_{\mathrm{C}}$ (both are denoted as $T_{\mathrm{t}}$ ) and the transition related magnetic entropy change $\left(\left|\triangle S_{\mathrm{m}}\right|_{\max }\right)$ for a field change of $2 \mathrm{~T}$ in some systems. Here we do not distinguish the structural and magnetic transitions as we now study the capacity of pressure to tune the transitions, which is associated with the tuning of related magnetocaloric effects. Among all referenced systems here, $\mathrm{MnNi}_{0.77} \mathrm{Fe}_{0.23} \mathrm{Ge}$ exhibits a prominent response of transition temperature with pressure. Therefore, for this alloy, it is easier to drive the martensitic transition by applying moderate hydrostatic pressures. Moreover, the large value of $d T_{\mathrm{t}} / d P$ indicates that the magnetostructural tran-
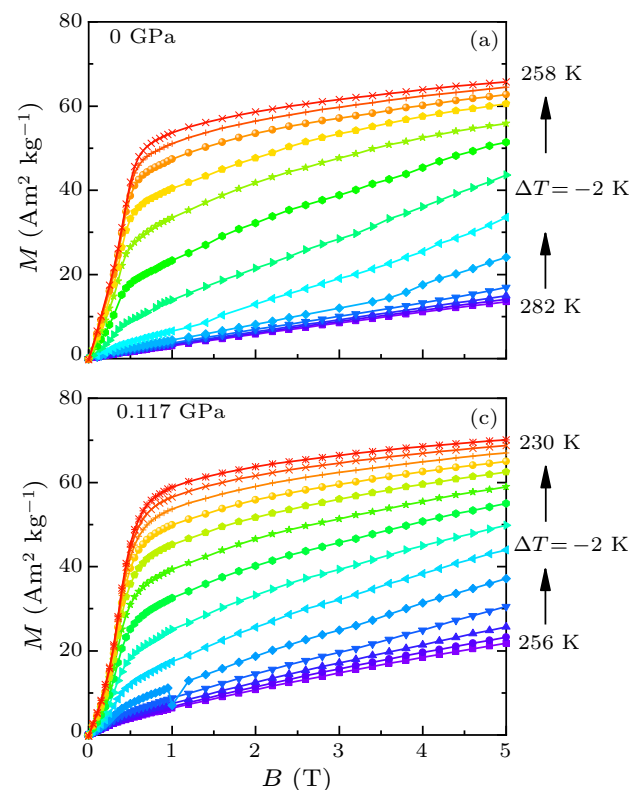

sition could be induced reversibly between the $T_{\mathrm{M}}$ at ambient pressure and the reverse martensitic transition temperature $\left(T_{\mathrm{A}}\right)$ at a proper pressure, for example, $0.117 \mathrm{GPa}$. Due to the above characteristic, $\mathrm{MnNi}_{0.77} \mathrm{Fe}_{0.23} \mathrm{Ge}$ as well as its related systems are proper platforms for research of barocaloric effect and applications. ${ }^{[36-38]}$
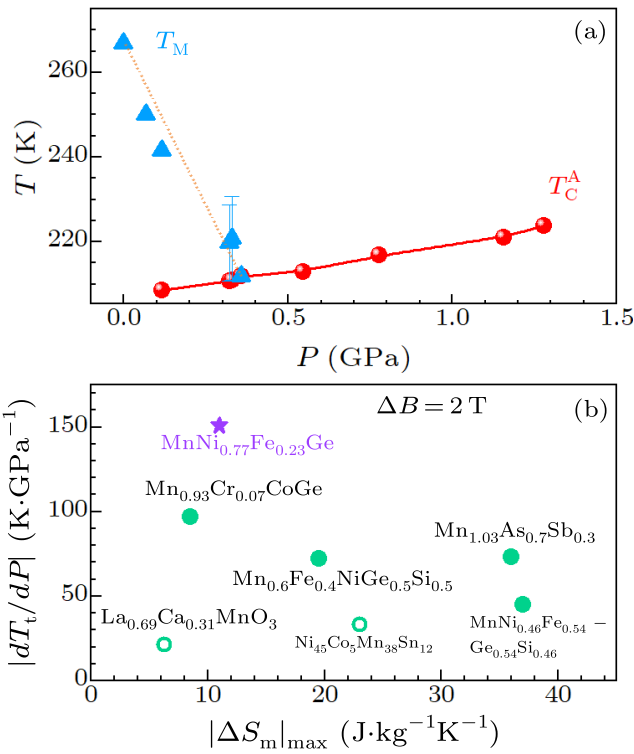

Fig. 2. (a) Pressure dependence of the martensitic transition temperature and Curie temperature of the parent phase. The orange dotted line is a guide for the eyes. (b) The value of $\left|d T_{\mathrm{t}} / d P\right|$ and $\left|\Delta S_{\mathrm{m}}\right|_{\max }$ (for a magnetic field change of $2 \mathrm{~T}$ ) in several systems, where the temperatures of structural and magnetic transitions are denoted as $T_{\mathrm{t}}$. The magnetic entropy changes are taken as the maximum values under different pressures for different systems. The solid symbols correspond to the negative $d T_{\mathrm{t}} / d P$ and $\triangle S_{\mathrm{m}}$ while the open symbols to the positive values. Data is taken from Refs. [11,17,19,21,33,34].
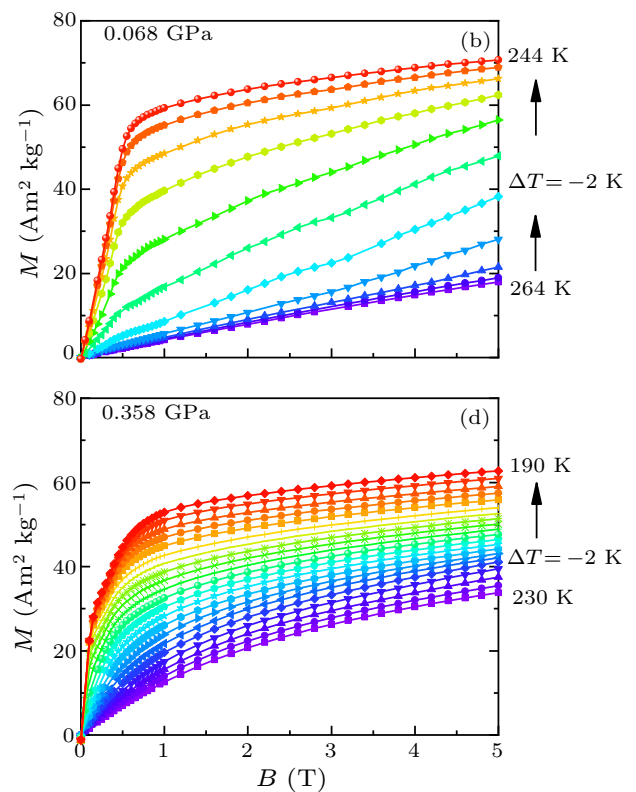

Fig. 3. Magnetic isothermals in a temperature interval of $-2 \mathrm{~K}$ measured under $0,0.068,0.117$ and $0.358 \mathrm{GPa}$. 
We performed magnetic isothermal measurements during cooling in a loop process ${ }^{[39]}$ to avoid the influence of the magnetization history. The magnetic isothermals with a temperature interval of $-2 \mathrm{~K}$ are shown in Fig. 3.

The study of magnetocaloric effect (MCE) in the $\mathrm{MnNi}_{0.77} \mathrm{Fe}_{0.23} \mathrm{Ge}$ system at ambient and under hydrostatic pressures was based on the Maxwell relation. ${ }^{[39]}$ The magnetic entropy change associated with the magnetic field variation can be expressed as

$$
\Delta S_{\mathrm{m}}(T, B)=\int_{0}^{B}\left(\frac{\partial S}{\partial B}\right)_{T} d B=\int_{0}^{B}\left(\frac{\partial M}{\partial T}\right)_{B} d B .(1)
$$

The magnetic field induced martensitic transformation in $\mathrm{MnNi}_{0.77} \mathrm{Fe}_{0.23} \mathrm{Ge}$ is not reversible, i.e., the martensite phase induced by magnetic field loading will not transform to parent phase totally during demagnetization process. Thus we concentrate on the process in which the parent phase transforms to the martensite phase driven by increasing magnetic field We calculated the magnetic entropy change of the magnetization process through Eq. (1) using magnetic isothermals data shown in Fig. 3.
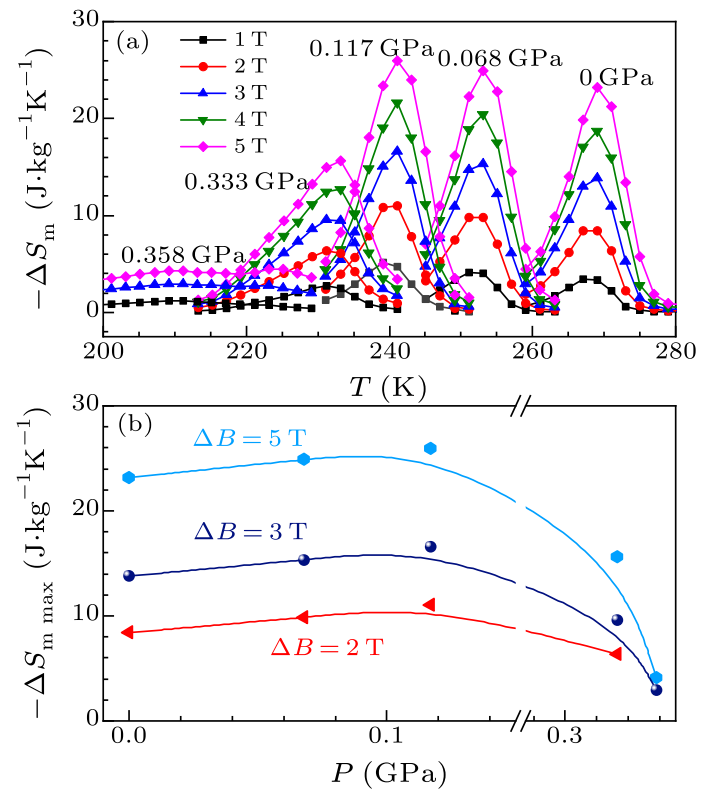

Fig. 4. (a) Temperature dependence of the magnetic entropy changes $\left(-\Delta S_{\mathrm{m}}\right)$ with magnetic field variation from 1 to $5 \mathrm{~T}$ under different pressures. (b) The maximum of the magnetic entropy changes $\left(-\Delta S_{m \text { max }}\right)$ under different pressures for a field change of $2 \mathrm{~T}, 3 \mathrm{~T}$ and $5 \mathrm{~T}$.

Figure 4(a) shows the entropy changes in several magnetic field variations under different pressures. The entropy change is reduced under $0.333 \mathrm{GPa}$ and approaches nearly zero under $0.358 \mathrm{GPa}$ due to the suppression of the martensitic transition by high pressures. However, the $\Delta S_{\mathrm{m}}$ reveals a nearly unchanged value in the range of about -23.2 to $-26.0 \mathrm{~J} \cdot \mathrm{kg}^{-1} \mathrm{~K}^{-1}$ for a field change of $5 \mathrm{~T}$ (Fig. 4(b)) over the pressure range from 0 to $0.117 \mathrm{GPa}$. The conserved and tunable
MCE under hydrostatic pressure can offer indications to the practical applications of caloric materials.

In summary, the study of magnetostructural transition and MCE under hydrostatic pressure in $\mathrm{MnNi}_{0.77} \mathrm{Fe}_{0.23} \mathrm{Ge}$ reveals that hydrostatic pressure can efficiently shift the magnetostructural transition temperature and thus the related MCE in this system. The decoupling of structural and magnetic phase transitions occurs at about $0.358 \mathrm{GPa}$, below which the MCE can be conserved and tuned by moderate pressures in a temperature range from 240 to $270 \mathrm{~K}$. This characteristic can be useful in practical applications as one can tune the MCE to the required temperature range by applying moderate pressures. More importantly, the remarkable ability to shift the magnetostructural transition temperature by hydrostatic pressure indicates a promising barocaloric effect driven by pressure loading around the magnetostructural phase transition in this alloy with a large volume change.

\section{References}

1] Liu E K et al. 2012 Nat. Commun. 3873

2] Sun A et al. 2015 Physica B 47427

3] Wei Z Y et al. 2015 Adv. Electron. Mater. 11500076

[4] Zhao Y Y et al. 2015 J. Am. Chem. Soc. 1371746

[5] Xu K et al. 2017 Sci. Rep. 741675

[6] Chen L et al. 2018 Sci. Chin. Phys. Mech. Astron. 61 056121

77 Franco V et al. 2018 Prog. Mater. Sci. 93112

[8] Liu R S et al. 2020 Chin. Phys. Lett. 37017501

[9] Li Y et al. 2016 APL Mater. 4071101

[10] Nizioł S et al. 1983 J. Magn. Magn. Mater. 38205

[11] Caron L, Trung N T and Brück E 2011 Phys. Rev. B 84 020414(R)

[12] Liu E K et al. 2010 Europhys. Lett. 9117003

[13] Li Y et al. 2019 Acta Mater. 174289

[14] Shen B G et al. 2009 Adv. Mater. 214545

[15] Moya X et al. 2013 Nat. Mater. 1252

[16] Fujita A et al. 2006 Phys. Rev. B 73104420

[17] Sun Y et al. 2006 Appl. Phys. Lett. 88102505

[18] Mañosa L S et al. 2008 Appl. Phys. Lett. 92012515

[19] Wada H, Matsuo S and Mitsuda A 2009 Phys. Rev. B 79 092407

[20] Kaštil J et al. 2015 J. Alloys Compd. 650248

[21] Samanta T et al. 2015 Phys. Rev. B $91020401(\mathrm{R})$

[22] Khalid S, Sabino F P and Janotti A 2018 Phys. Rev. B 98 220102

[23] Liu F et al. 2019 Sci. Chin. Phys. Mech. Astron. 6248211

[24] You W et al. 2019 Sci. Chin. Phys. Mech. Astron. 62 957411

[25] Jiang S et al. 2019 Chin. Phys. Lett. 36046103

[26] Shang Y X et al. 2019 Chin. Phys. Lett. 36086201

[27] Eiling A and Schilling J S 1981 J. Phys. F 11623

[28] Johnson V 1975 Inorg. Chem. 141117

[29] Bazela W et al. 1976 Phys. Status Solidi A 38721

[30] Fjellvåg H and Andresen A F 1985 J. Magn. Magn. Mater. 50291

[31] Bażela W et al. 1981 Phys. Status Solidi A 64367

[32] Liu E et al. 2011 IEEE Trans. Magn. 474041

[33] Nayak A K et al. 2009 J. Appl. Phys. 106053901

[34] Taubel A et al. 2017 J. Phys. D 50464005

[35] Kanomata T et al. 1995 J. Magn. Magn. Mater. 140-144 131

[36] Lloveras P et al. 2015 Nat. Commun. 68801

[37] Aznar A et al. 2019 Adv. Mater. 311903577

[38] Lloveras P et al. 2019 Nat. Commun. 101803

[39] Caron L et al. 2009 J. Magn. Magn. Mater. 3213559 